\title{
Técnica da Oscilação Longitudinal da Tocha para o Incremento da Produção na Soldagem em Ângulo entre Chapas de Espessuras Dissimilares
}

\author{
Nelso Gauze Bonacorso ${ }^{1}$, Adriano Albino Machado ${ }^{1}$, Rafael Pereira Pires ${ }^{1}$, Ivan Luiz Hubert ${ }^{1}$, Cassiano Bonin ${ }^{1}$, \\ Erwin Werner Teichmann ${ }^{1}$ \\ 1 Instituto Federal de Educação, Ciência e Tecnologia da Santa Catarina - IFSC, Departamento Acadêmico de Santa Catarina - \\ DAMM, Florianópolis, SC, Brasil.
}

Recebido: 23 Abr., 2017

Aceito: 02 Ago., 2017

E-mail: nelso@ifsc.edu.br (NGB)
Resumo: Soldagem em ângulo de componentes estruturais com espessuras dissimilares por tradicionais procedimentos, manuais ou automáticos, de soldagem podem apresentar falta de penetração, mordeduras e perfurações. A ocorrência destes defeitos se deve ao uso de uma incorreta corrente de soldagem e à falta de robustez do procedimento de soldagem diante das possíveis irregularidades geométricas nas juntas. Além disso, a profundidade das mordeduras e a quantidade de perfurações são ampliadas quando se deseja aumentar a produção. Isso se deve ao incremento da potência elétrica do arco para manter constante a quantidade de material depositado na junta em função do aumento da velocidade de soldagem. O presente artigo propõe o uso da técnica de oscilação longitudinal da tocha de soldagem, conhecida na literatura por switch back, para aumentar a produção com qualidade na soldagem. Foram usadas trajetórias de soldagem do tipo linear convencional e switch back de três passes com velocidade da tocha constante e variável (velocidade de avanço quatro vezes maior que a de retorno). O critério de aprovação dos cordões de solda foi baseado nas normas ISO de soldagem. Os resultados evidenciaram um aumento não significativo de produção com o uso da técnica switch back do tipo velocidade constante da tocha.

Palavras-chave: GMAW; Mecanização da soldagem; Junta em ângulo; Procedimento robusto; Switch back.

\section{Switch Back Technique for the Increase of the Production in the Angle Welding between Plates of Dissimilar Thicknesses}

\begin{abstract}
Angle welding of structural components with dissimilar thickness by traditional, manual or automatics, welding procedures can show lack of penetration, undercut and perforations. The occurrence of these defects must be the incorrect use of the welding current and the lack of robustness of the welding procedure among the geometrical irregularity possibilities in the joints. In addition, the depth of the undercut and the quantity of the perforations are enlarged when the increase of the production is wanted. This occurs due the electrical power increment from the arc to keep constant the quantity of the material in the welding joint to get higher the welding speed. This work apply the longitudinal oscillation from the welding torch known on the literature as switch back, to increase the production with quality in welding. Were used trajectories of the type linear conventional and switch back of three steps with constant and variable torch velocity (forward velocity four times faster than the return). The weld seams approval standard was based in the welding ISO. The results showed a non-significant increase of the production with the technique switch back of the type constant speed from the torch.
\end{abstract}

Key-words: GMAW; Mechanization welding; Joint in angle; Robust procedure; Switch back.

\section{Introdução}

Componentes estruturais unidos pelo processo GMAW, devem ter resistência mecânica adequada para suportar os esforços de trabalho, sem que haja rompimento das soldas de união. A qualidade das referidas uniões está diretamente relacionada com a corrente elétrica de soldagem usada, que deve ser diretamente proporcional à espessura dos componentes estruturais que formam a junta.

Nos casos em que os componentes estruturais têm a mesma espessura a corrente, a velocidade de soldagem a serem aplicadas e os outros parâmetros do procedimento estão, na maioria das situações, disponíveis em documentação técnica. Porém, em muitas 
situações práticas os componentes estruturais possuem espessuras diferenciadas. Nestes casos, os parâmetros do procedimento de soldagem são mais difíceis de serem encontrados, tanto na literatura, quanto determinados de forma experimental.

A Figura 1 mostra três situações de reprovação de uniões soldadas por procedimentos inadequados. Na Figura 1a ocorre o defeito de falta de penetração por uso de uma menor corrente elétrica e/ou uma maior velocidade de soldagem, enquanto que na Figura 1 b uma corrente elétrica de maior intensidade produziu uma acentuada mordedura no lado direito da chapa fina.

Em alguns casos ocorrem inícios de perfurações, Figura 1c, devido ao excesso de velocidade de soldagem que atrasa o deslocamento da poça de fusão em relação ao arco elétrico. Esse tipo de defeito na literatura é conhecido por humping [1].
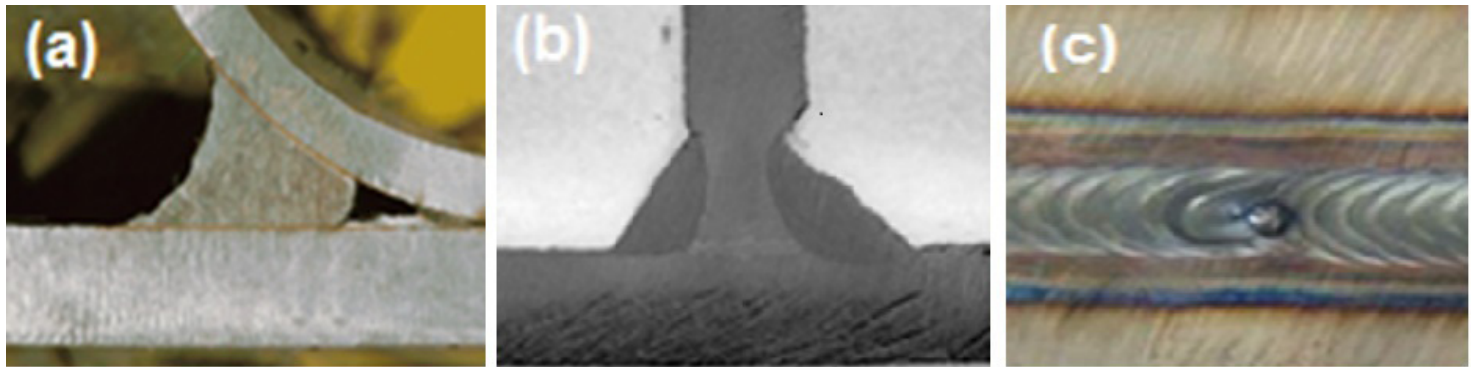

Figura 1. Defeitos de soldagem: (a) falta de penetração, (b) mordedura, (c) humping.

A técnica de oscilação longitudinal da tocha, conhecida como switch back, Figura 2, é usada por soldadores com o objetivo de alongar a poça de fusão e, com isso, minimizar mordeduras e perfurações na junta. Na soldagem automática, trabalhos obtiveram incrementos de produção com o uso da técnica switch back em comparação à soldagem linear convencional. Em ambos, foi usado o processo GMAW com corrente pulsada na soldagem de raiz de juntas de topo sem a utilização de cobre-junta [2,3].

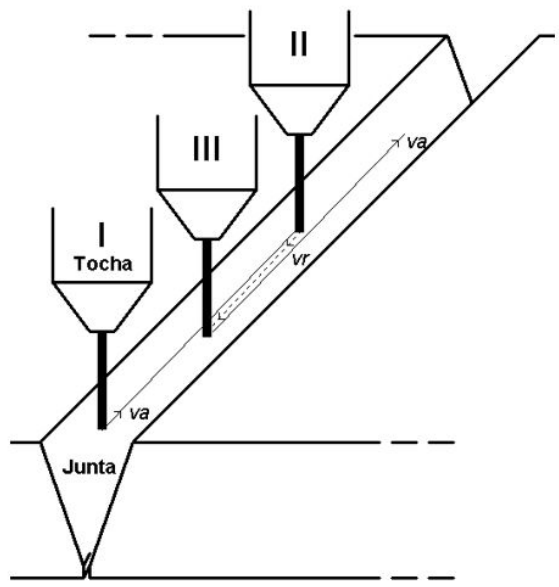

Figura 2. Ilustração da técnica switch back, com o avanço da tocha de I a II e retorno de II a III.

Também na soldagem automática, pesquisas revelaram um aumento da velocidade de soldagem em até $60 \%$ com o uso da técnica switch back [4,5]. Este aumento expressivo de produção foi obtido em juntas sobrepostas formadas por chapas de aço de $3 \mathrm{~mm}$ de espessura com o processo GMAW pulsado. Com o uso da trajetória linear convencional, a velocidade de soldagem máxima com aparência e penetração adequados dos 
Técnica da Oscilação Longitudinal da Tocha para o Incremento da Produção na Soldagem em Ângulo entre Chapas de Espessuras Dissimilares

cordões de solda foi de $0,35 \mathrm{~m} / \mathrm{min}$, enquanto que com a técnica switch back a velocidade de soldagem máxima alcançada foi de $0,57 \mathrm{~m} / \mathrm{min}$.

Com base nos bons resultados produzidos pelas pesquisas citadas, esse artigo tem como objetivo principal usar a técnica switch back em comparação com a linear convencional para determinar o percentual do aumento de produção com qualidade na soldagem automática em ângulo entre chapas de espessuras dissimilares.

\section{Materiais e Métodos}

Para atingir o objetivo supracitado, corpos de prova foram construídos como ilustra a Figura 3 , a partir de chapas planas de aço carbono 1020 cortadas em duas espessuras diferentes: 6,35 mm e 2,00 mm. A junta a ser soldada é em ângulo do tipo " $T$ ", formada pela interseção da chapa mais espessa vertical com a chapa menos espessa horizontal.

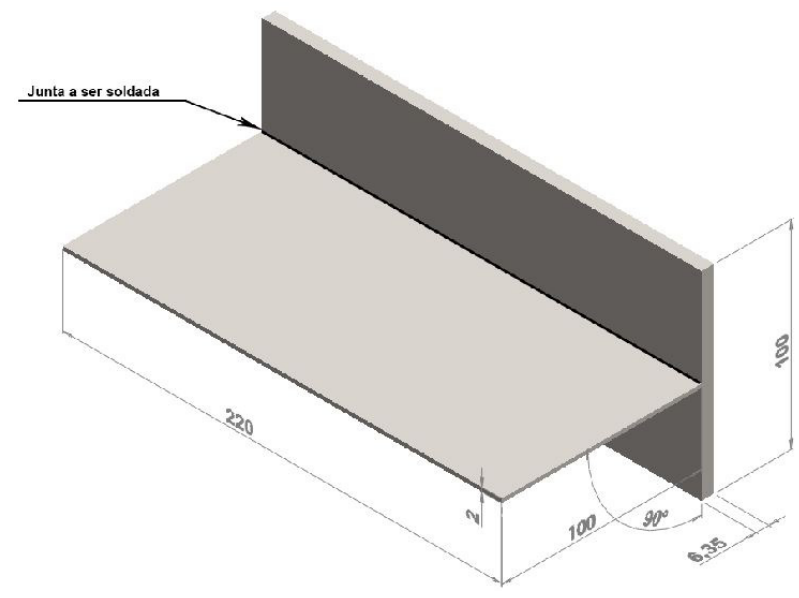

Figura 3. Características geométricas dos corpos de prova.

O arame AWS ER70S-6, de 1,0 mm de diâmetro foi o material de adição usado para soldar os corpos de prova. $O$ gás de proteção usado foi uma mistura gasosa, $98 \%$ de argônio e $2 \%$ de oxigênio, com vazão de $15 \mathrm{l} / \mathrm{min}$. Esta mistura foi escolhida em razão de garantir uma transferência metálica por spray no processo GMAW a partir da corrente de transição.

A fonte de soldagem, Figura 4a, foi configura para o processo GMAW em modo corrente contínua e na polaridade positiva. No primeiro eixo, trilho linear $X$, da cadeia cinemática do manipulador de soldagem, Figura 4b, foi fixado por parafusos o dispositivo com ajuste mecânico dos outros três graus de liberdade (um linear $Z$ e dois angulares: ao redor dos eixos X e Y), Figura 5a. Por sua vez, a tocha GMAW foi fixada na extremidade do referido dispositivo, Figura 4b.
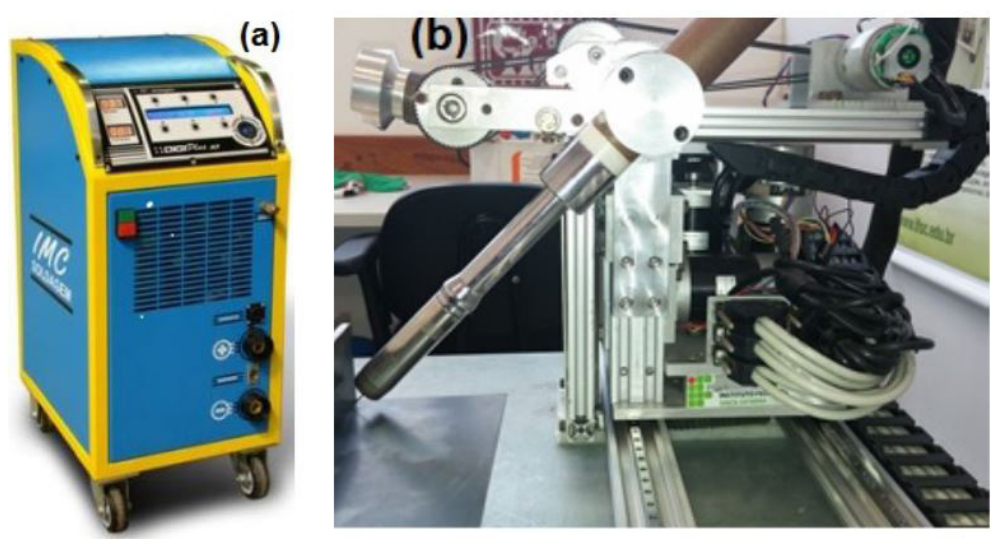

Figura 4. Equipamentos de soldagem: (a) Fonte multiprocesso IMC, (b) Manipulador de soldagem IFSC. 


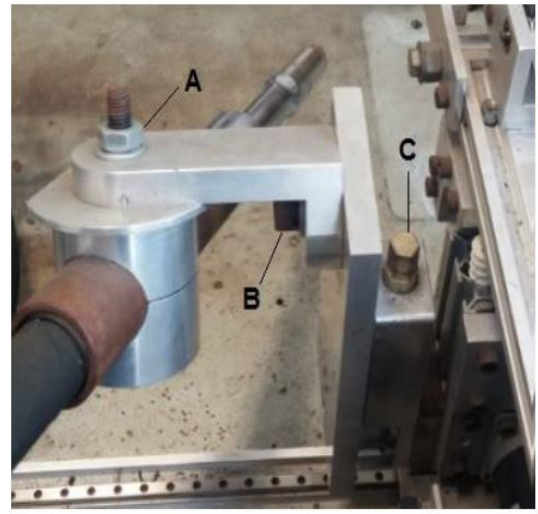

(a)

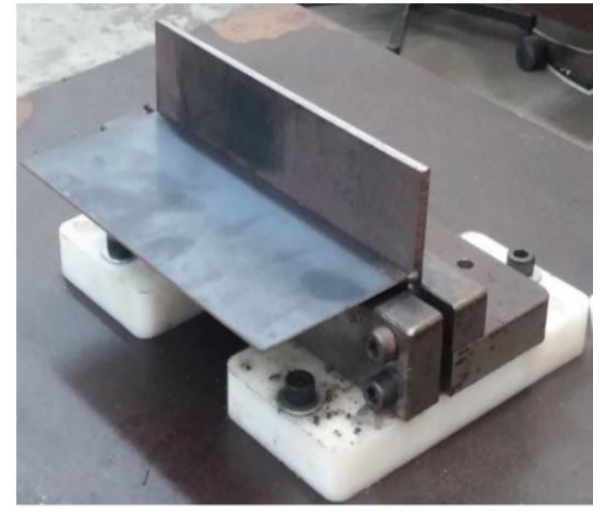

(b)

Figura 5. (a) Dispositivo com os ajustes dos três graus de liberdade: ângulo de ataque em $A$, ângulo de sentido de soldagem em B e deslocamento vertical em C; (b) Morsa para fixação dos corpos de prova.

A inserção desse dispositivo de três graus de liberdade, Figura $5 a$, e da morsa de fixação dos corpos de prova, Figura $5 b$, foram medidas preventivas para evitar respectivamente a falta de rigidez do manipulador de soldagem e as deformações nos corpos de prova durante a soldagem. Como a junta a ser soldada é paralela ao trilho são necessários apenas quatro graus de liberdade para definir a posição e a orientação da tocha em relação à junta a ser soldada. No manipulador foram programados, em referencia ao seu eixo $\mathrm{X}$, os pontos de inicio e de fim da junta, a velocidade de soldagem e a parametrização de cada tipo de trajetória: linear convencional ou switch back. No dispositivo foram ajustados mecanicamente via parafusos o afastamento vertical em Z da ponta do arame em relação ao vértice da junta, o ângulo de sentido de soldagem (ao redor do eixo X) e o ângulo de ataque (ao redor do eixo $\mathrm{Y}$ ).

A metodologia aplicada foi estruturada em uma sequência de quatro etapas: cálculo do valor da constante da relação entre a velocidade de alimentação do arame e a velocidade média de soldagem; determinação experimental da corrente de transição e da equação matemática da corrente elétrica de soldagem em função da velocidade de alimentação do arame ( $i=f(V a))$; obtenção por experimentos do posicionamento e da orientação da tocha de solda em relação à junta do corpo de prova e a determinação via ensaios das velocidades de soldagem máximas para as trajetórias, linear convencional e switch back com velocidade da tocha constante e variável.

Por sua vez, a obtenção do valor da velocidade de soldagem máxima está vinculada a aparência visual adequada do cordão de solda e aos oito parâmetros dimensionais referentes à secção transversal, Figura 6, com seus respectivos critérios de qualidade, Tabela 1, baseados nas normas ISO de soldagem.

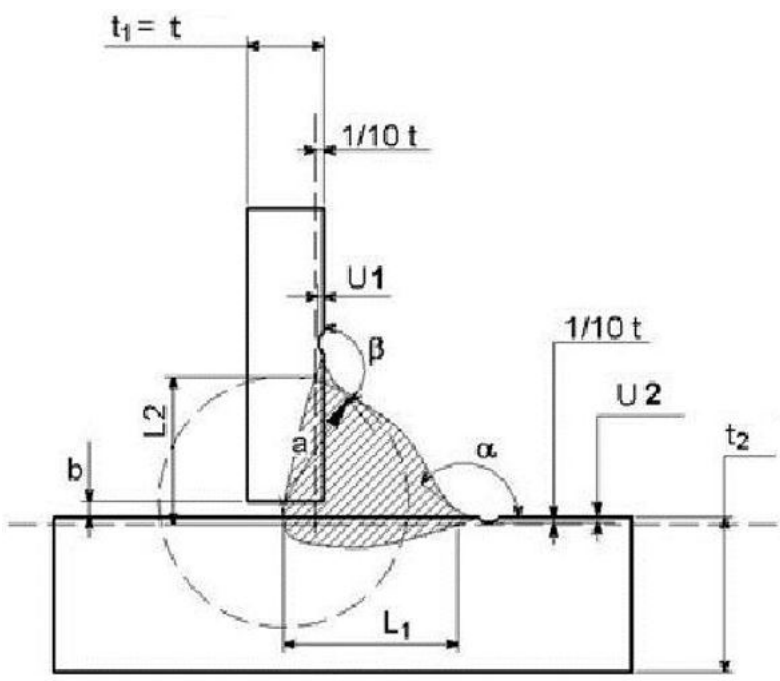

Figura 6. Parâmetros relacionados pelas normas ISO com base na espessura da chapa mais fina, $\mathrm{t}_{1}$. 
Técnica da Oscilação Longitudinal da Tocha para o Incremento da Produção na Soldagem em Ângulo entre Chapas de Espessuras Dissimilares

Tabela 1. Valores admissíveis aplicados aos critérios de qualidade da solda de união.

\begin{tabular}{cc} 
Parâmetros & Critérios de Qualidade e Faixas Toleráveis \\
Mordeduras $(\mathrm{mm})$ & $\mathrm{U}_{1} \leq 0,1 . \mathrm{t}_{1} \rightarrow \mathrm{U}_{1} \leq 0,20$ e $\mathrm{U}_{2} \leq 0,1 . \mathrm{t}_{2} \rightarrow \mathrm{U}_{2} \leq 0,64$ \\
Penetrações $(\mathrm{mm})$ & $\mathrm{L}_{1}=\mathrm{L}_{2}>0,8 . \mathrm{t} \rightarrow \mathrm{L}_{1}=\mathrm{L}_{2}>1,60$ \\
Garganta $(\mathrm{mm})$ & $\mathrm{a} \geq 0,8 . \mathrm{t} \rightarrow \mathrm{a} \geq 1,60$ \\
Espaçamento $(\mathrm{mm})$ & $\mathrm{b} \leq 0,2 . \mathrm{t} \rightarrow \mathrm{b} \leq 0,40$ \\
Ângulos entre o cordão e a peça $\left({ }^{\circ}\right)$ & $a \geq 110$ e $\beta \geq 110$ \\
\hline
\end{tabular}

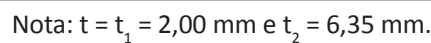

Na técnica switch back de três passes sobre cada segmento da junta, Figura 2, a tocha avança, da posição I para a II, uma distância igual à da com velocidade va e, na sequência recua, da posição II para a III, uma distância igual a $d r=d a / 2 \mathrm{com}$ velocidade $v r$. O período de cada ciclo $T$ é constituído pela soma de duas parcelas, o tempo de avanço ta e o tempo de retorno da tocha tr. O fator adimensional $D$ conhecido por razão cíclica é definido pela razão entre o tempo de avanço ta e o período $T$, Equação 1:

$$
D=\frac{t a}{T}=\frac{t a}{t a+t r}, \text { onde } 0<D<1
$$

Neste tipo de trajetória switch back, a velocidade média de soldagem é definida pela Equação 2:

$$
V s_{m}=\frac{d r}{T}=\frac{d a}{2 . T}
$$

Assim, as velocidades de avanço va e de retorno vr da tocha, Equação 3, são expressas em função da velocidade de soldagem $V s_{m}$ e da razão cíclica $D$ :

$$
v a=\frac{d a}{t a}=\frac{2 \cdot V s_{m}}{D} \text { e } v r=\frac{d r}{t r}=\frac{V s_{m}}{1-D}
$$

No caso que se deseja velocidade da tocha constante, basta igualar $v a=v r$ na Equação 3 para obter $D=2 / 3 \mathrm{e}$, na sequência, obter: $v a=v r=3 . V s_{m}$. Para os casos onde se deseja velocidades de avanço e de retorno diferenciadas segue-se o mesmo raciocínio. Uma situação investigada nesse trabalho foi o uso de $v a=4$.vr. Nesta situação, $D=1 / 3$ e as relações de velocidades resultam em: $v a=6 . V s_{m}$ e $v r=1,5 . V s_{m}$.

\section{Resultados e Discussão}

A velocidade média de soldagem de $0,50 \mathrm{~m} / \mathrm{min}$ foi utilizada como valor inicial dos experimentos com base na medição da velocidade de soldagem de soldadores experientes para este tipo de junta [6]. Para definir a quantidade ideal de material a ser depositado na junta foi considerado um triângulo isósceles, com seus lados iguais de comprimento $5 \mathrm{~mm}$ voltados para as chapas que constituem a junta, como sendo a forma geométrica da área depositada. Embora $5 \mathrm{~mm}$ seja bem maior do que o limite mínimo das penetrações aceito pelas normas ISO, Figura 6, esse valor foi selecionado com o objetivo de aumentar a largura do cordão de solda para minimizar a ocorrência das mordeduras nas chapas que compõem a junta.

Com a área de $12,50 \mathrm{~mm}^{2}$ do triângulo isósceles e a velocidade de soldagem mínima de $0,50 \mathrm{~m} / \mathrm{min}$, obteve-se o volume de material depositado de $6250 \mathrm{~mm}^{3} \mathrm{em} 1$ minuto de arco aberto. Dividindo esse volume pela área de $0,78 \mathrm{~mm}^{2}$ correspondente a seção transversal do arame de bitola $1 \mathrm{~mm}$ usado, obteve-se o comprimento de arame de 7958,75 mm, ou seja, $8 \mathrm{~m} / \mathrm{min}$ de velocidade do arame. Assim, foi definido o valor de 16 para a constante que define a razão entre a velocidade de alimentação do arame $V a$ e a velocidade média de soldagem $V s_{m^{\prime}}$ ou seja:

$$
V a=16 . V s_{m}
$$


A seguir foram realizados experimentos de soldagem robotizada sobre chapas planas de aço carbono 1020 de espessura 6,35 mm, Figura 7, para obter o valor da corrente de transição para o modo spray correspondente à velocidade de arame mínima de $8 \mathrm{~m} / \mathrm{min}$. Para uma distância bico de contato peça (DBCP) de $20 \mathrm{~mm}$ e comprimento de arco em torno de $5 \mathrm{~mm}$ foi ajustado o valor de $172 \mathrm{~A}$ com a fonte de soldagem operando em modo corrente. Na sequência foram realizados ensaios para ajustar mais dois pontos de funcionamento, $P_{k}(\mathrm{Va}, i)$, com o objetivo de obter o polinômio de grau dois da corrente elétrica de soldagem em função da velocidade de arame, Equação 5:

$$
i=f(V a)=a_{0} \cdot V a^{2}+a_{1} \cdot V a+a_{2}
$$

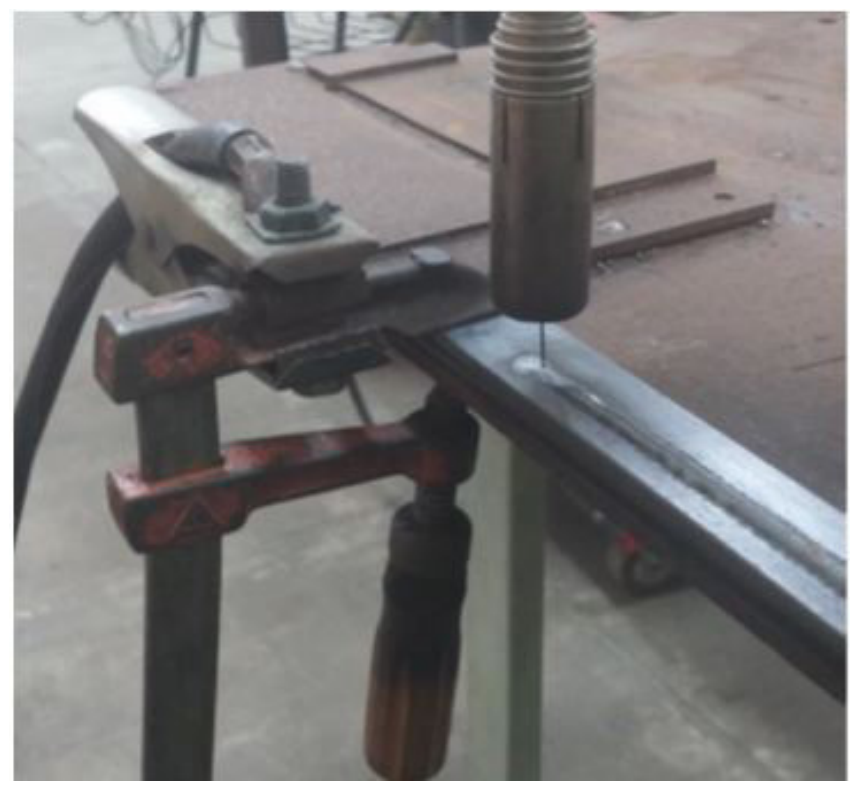

Figura 7. Foto do ensaio GMAW robotizado para determinar os pontos $P_{k}(V a ; i)$, com $k=1,2$ e 3.

O método matemático dos mínimos quadrados foi aplicado nos pontos $\left(P_{1}(8,0 ; 172), P_{2}(12,0 ; 220)\right.$ e $\left.P_{3}(16,0 ; 268)\right)$ para determinar os coeficientes $\left(a_{0}=0 ; a_{1}=12\right.$ e $\left.a_{3}=76\right)$ da Equação 5. Assim, a parametrização da fonte de soldagem ( Va e $i$ ), obtida via o uso sequencial das Equações 4 e 5 ficou só dependente da velocidade média de soldagem $V s_{m}$. Em função da velocidade máxima do alimentador automático de arame, $V a_{\text {máx }}=20 \mathrm{~m} / \mathrm{min}$, ficou viável realizar uniões com transferência metálica por spray em uma faixa de velocidade média de soldagem de 0,50 $\mathrm{m} / \mathrm{min}$ até $1,25 \mathrm{~m} / \mathrm{min}$.

Na sequência foi determinado via análise das macrografias dos corpos de prova soldados com velocidade de 0,50 $\mathrm{m} / \mathrm{min}$, o ajuste dos três graus de liberdade do dispositivo mecânico de posicionamento vertical e de orientação da tocha que são o ângulo de sentido de soldagem e o ângulo de ataque.

Com o objetivo de determinar o correto valor do ângulo no sentido de soldagem foi mantido nulo o ângulo de ataque e a ponta do arame foi direcionada para o vértice da junta. O valor inicial foi de 45 graus. A cada experimento este ângulo foi incrementado em 5 graus. Constatou-se que o valor de $60^{\circ}$ produzia uma deposição semelhante nas duas chapas, porém com uma mordedura mais acentuada na chapa fina. A solução encontrada que resolveu este problema foi a de afastar verticalmente em $1 \mathrm{~mm}$ a ponta do arame em relação ao vértice da junta, lado direito da Figura 8.

A seguir, foram realizados ensaios de soldagem, conforme ilustrado no lado direito da Figura 8, com o objetivo de definir o ângulo de ataque para minimizar as mordeduras e as penetrações nas chapas. 0 valor inicial foi o de 5 graus empurrando. A cada experimento este ângulo foi incrementado de 5 graus. 0 valor de 20 graus, lado esquerdo da Figura 8, produziu o melhor resultado com base nas normas ISO de soldagem, Tabela 1.

Após definidas as condições ideais de orientação e posicionamento da tocha para a soldagem dos corpos de prova realizou-se os experimentos com a estratégia da trajetória linear convencional da tocha de soldagem. O intervalo de experimentos com esta estratégia iniciou-se com a velocidade de 0,5 m/min. Para cada novo 
Técnica da Oscilação Longitudinal da Tocha para o Incremento da Produção na Soldagem em Ângulo entre Chapas de Espessuras Dissimilares

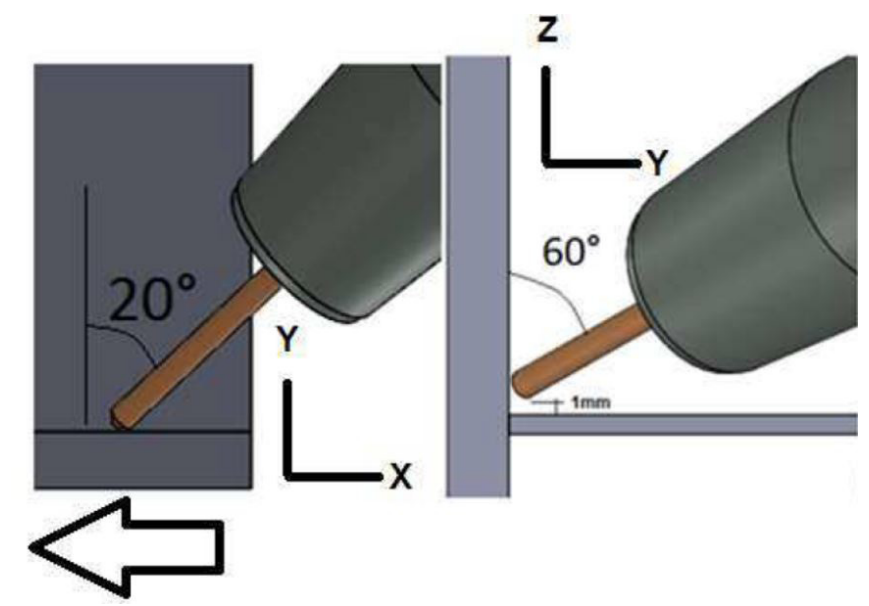

Figura 8. Ângulo de ataque à esquerda e o ângulo de sentido de soldagem e o afastamento vertical à direita.

experimento realizado foi incrementado $0,1 \mathrm{~m} / \mathrm{min}$ na velocidade de soldagem. A Tabela 2 apresenta os resultados obtidos com a trajetória linear convencional. Nesta estão descritos, para cada união realizada: a velocidade de soldagem usada, os valores medidos de tensão e de corrente elétrica média, a energia de soldagem calculada por centímetro, a macrografia e os valores medidos de seus oitos parâmetros de acordo com a Figura 6.

A sexta união com velocidade de soldagem de $1,00 \mathrm{~m} / \mathrm{min}$ foi reprovada, como observado na Tabela 2 , devido à mordedura da chapa fina de 0,39 $\mathrm{mm}$ superar o valor de $0,20 \mathrm{~mm}$ estipulado por um dos critérios das normas ISO. As uniões aprovadas foram da primeira com velocidade de soldagem de $0,5 \mathrm{~m} / \mathrm{min}$ à quinta com $0,9 \mathrm{~m} / \mathrm{min}$.

Tabela 2. Resultados de soldagem com a trajetória do tipo linear convencional.

\begin{tabular}{|c|c|c|c|c|c|c|}
\hline \multirow{2}{*}{$\begin{array}{l}\text { Parâmetros e } \\
\text { Secções } \\
\text { Transversais }\end{array}$} & \multicolumn{6}{|c|}{ Experimentos } \\
\hline & União 1 & União 2 & União 3 & União 4 & União 5 & União 6 \\
\hline $\mathrm{Vs}_{\mathrm{m}}(\mathrm{m} / \mathrm{min})$ & 0,50 & 0,60 & 0,70 & 0,80 & 0,90 & 1,00 \\
\hline $\operatorname{Im}(\mathrm{A}) ; \operatorname{Vm}(\mathrm{V})$ & $170 ; 25,0$ & $187 ; 25,8$ & $206 ; 27,5$ & $226 ; 29,3$ & $245 ; 30,6$ & $263 ; 31,3$ \\
\hline $\mathrm{H}(\mathrm{kJ} / \mathrm{cm})$ & 5,10 & 4,82 & 4,86 & 4,97 & 5,00 & 4,94 \\
\hline \multicolumn{7}{|l|}{ Macros } \\
\hline $\mathrm{a} ; \mathrm{b}(\mathrm{mm})$ & 4,$25 ; 0,01$ & 4,$68 ; 0,05$ & 4,$92 ; 0,01$ & 4,$70 ; 0,01$ & 4,$85 ; 0,07$ & 4,$50 ; 0,08$ \\
\hline L1 ; L2 (mm) & 4,$13 ; 6,18$ & 4,$22 ; 5,55$ & 4,$34 ; 5,09$ & 4,$89 ; 5,21$ & 4,$56 ; 5,07$ & 4,$94 ; 4,57$ \\
\hline U1 ; U2 (mm) & 0,$00 ; 0,36$ & 0,$10 ; 0,26$ & 0,$21 ; 0,60$ & 0,$14 ; 0,48$ & 0,$17 ; 0,59$ & 0,$39 ; 0,33$ \\
\hline$\alpha ; \beta\left(^{0}\right)$ & $128 ; 138$ & $125 ; 128$ & $114 ; 118$ & $124 ; 128$ & $121 ; 120$ & $117 ; 138$ \\
\hline
\end{tabular}

Esta última união aprovada teve os valores de ambas às mordeduras muito próximas da reprovação. Assim, essa velocidade de soldagem foi considerada o limite de produção para a trajetória linear convencional.

O aumento progressivo das mordeduras em função do aumento da velocidade de soldagem e da correspondente potência do arco elétrico pode estar relacionado, como descrito em literatura, com o aumento da corrente em consequência da força magnética na região da solda e também pelo aumento na velocidade de propagação das partículas expelidas na transferência metálica do tipo spray.

Os valores referentes à energia de soldagem calculada por centímetro para cada união, Tabela 2, se apresentam muito próximos. No entanto, observa-se que há uma tendência ao aumento da mordedura e da penetração na junta. Este resultado evidencia o supracitado a respeito da relação entre o aumento da potência elétrica de soldagem com o incremento na mordedura e na penetração. 
As macrografias dos corpos de prova apresentadas na Tabela 2 revelam que o aumento da velocidade alterou significativamente a geometria do cordão. Além disso, houve uma distribuição mais uniforme do material de deposição, visto que, os comprimentos das penetrações, L1 e L2, apresentam dimensões cada vez mais próximas com o aumento da velocidade de soldagem.

Para determinar a velocidade limite para os dois tipos de trajetória do tipo switch back foram soldados corpos de prova com avanço de $10 \mathrm{~mm}$, retorno de $5 \mathrm{~mm}$ e velocidade de soldagem a partir de 0,90 $\mathrm{m} / \mathrm{min}$, Tabela 3 , seguida da análise dos critérios de aprovação da Tabela 1.

$\mathrm{Na}$ técnica switch back com velocidade constante as uniões foram aprovadas até a velocidade média de soldagem de $0,95 \mathrm{~m} / \mathrm{min}$. Ao aumentar a velocidade de soldagem para $1,00 \mathrm{~m} / \mathrm{min}$ a mordedura $U_{1}$ e o ângulo $\beta$ ultrapassaram o faixa admissível, reprovando assim a união.

Tabela 3. Resultados de soldagem com trajetórias do tipo switch back.

\begin{tabular}{|c|c|c|c|c|c|c|}
\hline \multirow{2}{*}{$\begin{array}{l}\text { Parâmetros e } \\
\text { Secções } \\
\text { Transversais }\end{array}$} & \multicolumn{3}{|c|}{ Experimentos com va $=\mathbf{v r}=3 . \mathrm{Vs}_{\mathrm{m}}$} & \multicolumn{3}{|c|}{ Experimentos com va $=4 . \mathrm{vr}=6 . \mathrm{Vs}_{\mathrm{m}}$} \\
\hline & União 7 & União 8 & União 9 & União 10 & União 11 & União 12 \\
\hline $\mathrm{Vs}_{\mathrm{m}}(\mathrm{m} / \mathrm{min})$ & 0,90 & 0,95 & 1,00 & 0,90 & 0,95 & 1,00 \\
\hline $\operatorname{Im}(\mathrm{A}) ; \mathrm{Vm}(\mathrm{V})$ & $246 ; 29,7$ & $261 ; 31,1$ & $263 ; 28,4$ & $246 ; 29,0$ & $261 ; 29,4$ & $263 ; 29,5$ \\
\hline $\mathrm{H}(\mathrm{kJ} / \mathrm{cm})$ & 4,87 & 5,14 & 4,47 & 4,76 & 4,86 & 4,66 \\
\hline \multicolumn{7}{|l|}{ Macros } \\
\hline $\mathrm{a} ; \mathrm{b}(\mathrm{mm})$ & 4,$74 ; 0,12$ & 4,$55 ; 0,02$ & 4,$24 ; 0,15$ & 4,$18 ; 0,30$ & 5,$20 ; 0,05$ & 4,$42 ; 0,18$ \\
\hline $\mathrm{L} 1 ; \mathrm{L} 2(\mathrm{~mm})$ & 4,$34 ; 5,65$ & 4,$52 ; 4,98$ & 4,$53 ; 4,40$ & 4,$73 ; 4,47$ & 4,$60 ; 4,20$ & 4,$80 ; 4,58$ \\
\hline U1 ; U2 (mm) & 0,$17 ; 0,34$ & 0,$09 ; 0,37$ & 0,$25 ; 0,15$ & 0,$13 ; 0,31$ & 0,$14 ; 0,59$ & 0,$33 ; 0,16$ \\
\hline$a ; \beta\left(^{0}\right)$ & $130 ; 126$ & $120 ; 120$ & $119 ; \mathbf{1 0 8}$ & $125 ; 124$ & $125 ; \mathbf{1 0 2}$ & $124 ; 117$ \\
\hline
\end{tabular}

Na técnica switch back com velocidade variável as uniões foram aprovadas até a velocidade média de soldagem de $0,90 \mathrm{~m} / \mathrm{min}$. Ao aumentar a velocidade de soldagem média para $0,95 \mathrm{~m} / \mathrm{min}$ o ângulo $\beta$ ultrapassou a faixa limite, reprovando assim a união. Aumentando ainda mais a velocidade de soldagem média para $1,00 \mathrm{~m} / \mathrm{min}$ a mordedura $U_{1}$ ultrapassou o valor admissível, reprovando também está união.

Assim, a técnica switch back com velocidade constante da tocha permitiu uma velocidade de soldagem limite um pouco maior do que a técnica switch back com velocidade variável da tocha. Como no caso da soldagem com velocidade linear convencional, os valores calculados referentes às respectivas energias de soldagem por centímetro usadas em cada união da Tabela 3 são próximos.

Os resultados obtidos demonstram que o ganho de velocidade de soldagem foi muito pequeno (cerca de $5 \%$ ) com o uso da técnica switch back em relação à convencional condução linear da tocha. Esta constatação diverge da opinião dos pesquisadores citados neste artigo [4-5], onde foram obtidos ganhos maiores do que dez vezes ao alcançado por este trabalho. Apesar do baixo ganho obtido neste trabalho, a velocidade de soldagem limite para a trajetória linear convencional foi muito superior a velocidade de soldagem usada para as trajetórias do tipo switch back em todos os trabalhos referenciados por este artigo.

Essa diferença é atribuída ao parâmetro ângulo de ataque que neste artigo foi sempre de $20^{\circ}$ empurrando para ambos os tipos de trajetória e nulo em todos os outros trabalhos referenciados. Da literatura de soldagem e também da aplicação industrial sabe-se que a soldagem empurrando na convencional condução linear da tocha proporciona um cordão de solda mais largo e com menor penetração e convexidade. Nesta condição, a pressão do arco empurra a poça de fusão no sentido da soldagem, minimizando assim, o surgimento das mordeduras. Isso é o resultado do arco elétrico atuar mais sobre o material líquido da poça de fusão do que nos metais de base formadores da junta. 
Técnica da Oscilação Longitudinal da Tocha para o Incremento da Produção na Soldagem em Ângulo entre Chapas de Espessuras Dissimilares

Portanto, não se obteve um ganho significativo de produção neste trabalho com a técnica switch back porque o efeito do ângulo de ataque empurrando a poça de fusão na trajetória linear convencional é tão positivo para o aumento da produção, quanto ao alongamento da poça de fusão proporcionada por uma melhor distribuição do calor do arco elétrico sobre a junta na técnica switch back. Apesar de também usar o mesmo valor do ângulo de ataque na técnica switch back a soma dos efeitos não foi verificada no sentido de aumentar a velocidade de soldagem. Talvez seja devido o ângulo de ataque mudar para puxando na etapa de retorno da tocha, proporcionando características opostas às supracitadas com ângulo de ataque empurrando.

Ainda comparando, a Figura 9 ilustra a aparência visual dos cordões de solda gerados com a mesma velocidade média de soldagem, 0,90 m/min, nos três tipos de trajetória usada. Para essa velocidade média de soldagem, a utilização das estratégias switch back na soldagem de juntas em comparação com a estratégia de soldagem linear convencional proporcionou a redução dos valores das mordeduras e o incremento dos ângulos entre o cordão de solda e a peça.

(a)

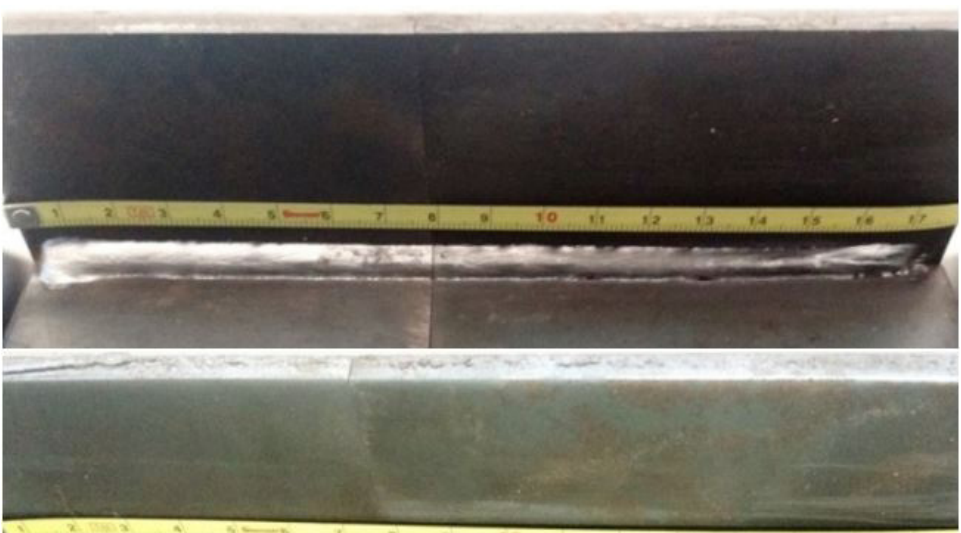

(b)

(c)

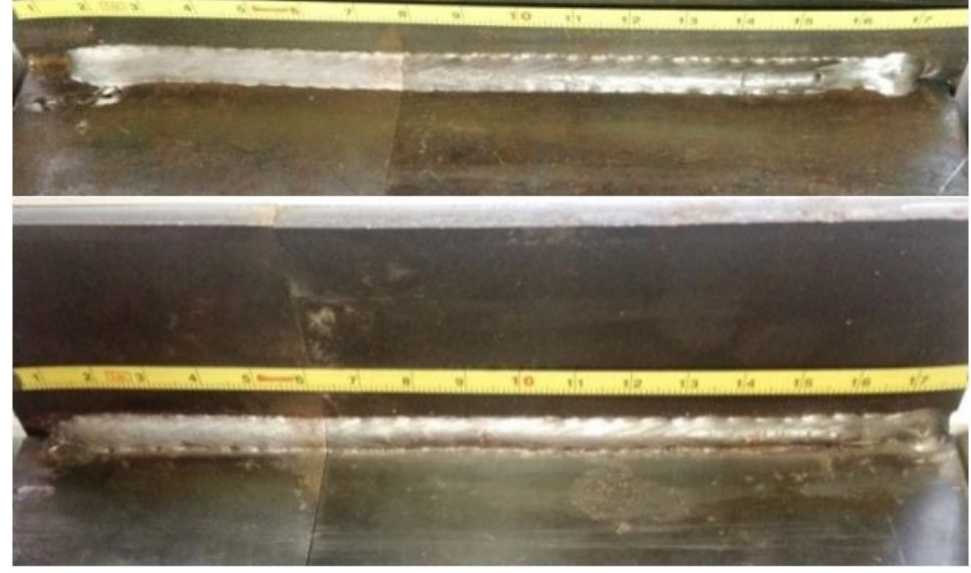

Figura 9. Aspecto visual das uniões com $V s_{m}=0,90 \mathrm{~m} / \mathrm{min}$, para as trajetórias: (a) linear convencional, (b) switch back com $v a=v r=3 . V s_{m}$ e (c) switch back com $v a=6 . V s_{m}$ e $v r=1,5 . V s_{m}$.

\section{Conclusões}

Com base nos resultados obtidos, pode-se afirmar que não houve aumento significativo da produção com o uso da técnica switch back nas condições experimentais descritas nesse artigo para a união em ângulo entre chapas de espessuras dissimilares. Somente a técnica switch back com velocidade constante teve um ganho de produção de aproximadamente $5 \%$ em relação à trajetória linear convencional. Cabe frisar que no expressivo ganho de produção de $60 \%$ obtido por Bruecker [4] e publicado por Almeida et al. [5] não foram aplicados critérios de qualidade para aprovação das uniões e a quantidade de material depositado na junta de sobrepor não era incrementada de forma proporcional com o aumento da velocidade de soldagem. Mesmo assim, o incremento 
obtido na velocidade de soldagem para ambos os tipos de trajetória neste artigo foram muito superiores. De $0,35 \mathrm{~m} / \mathrm{min}$ para $0,90 \mathrm{~m} / \mathrm{min}$ para a trajetória linear convencional e de $0,57 \mathrm{~m} / \mathrm{min}$ para $0,95 \mathrm{~m} / \mathrm{min}$ para a trajetória switch back com velocidade de avanço da tocha igual à de retorno. Portanto, fica evidenciado por este artigo que para incrementar a velocidade de soldagem é necessário modificar o ângulo de ataque para a condição de empurrar a poça de fusão. Essa constatação é baseada nos resultados obtidos pelos pesquisadores [2-5], que em seus experimentos mantiveram nulo o ângulo de ataque e, com isso, obtiveram uma menor produção.

\section{Agradecimentos}

Os autores agradecem ao DAMM do IFSC pela infraestrutura cedida e ao Programa de Apoio ao Fortalecimento dos Grupos de Pesquisa do IFSC pelo suporte financeiro.

\section{Referências}

[1] Nguyen TC, Weckman DC, Johnson DA, Kerr HW. High speed fusion weld bead defects. Science and Technology of Welding and Joining. 2006;11(6):618-633. http://dx.doi. org/10.1179/174329306X128464.

[2] Kaneko Y, Yamane S, Oshima K. Numerical simulation of MIG weld pool in switchback welding. Welding in the World. 2009;53(11/12):R333-R341. http://dx.doi.org/10.1007/ BF03263476.

[3] Yamane S, Uji K, Nakajima T, Yamamoto H. Application of switch back welding to $\mathrm{V}$ groove MAG welding. Welding International. 2015;29(2):103-109. http://dx.doi.org/10.1080/09507116.2012.753253.
[4] Bruecker D. Increasing travel speed with pulsed MIG switch-back welding on lap joint. Uberlândia: LAPROSOLDA/UFU; 2007. 16 p. Relatório Interno LAPROSOLDA/UFU 15.

[5] Almeida HAL, Mota CAM, Scotti A. Efeitos do comprimento de reversão e do ângulo de ataque sobre a estrutura de solidificação do cordão em soldagem MIG/MAG com "Switch Back". Soldagem \& Inspeção. 2012;17(2):123-137. http://dx.doi. org/10.1590/S0104-92242012000200006.

[6] Dutra JC, et al. Automating a wheel manufacturing operation. Welding Journal. 2014;93:76-84. 